\title{
Asymptotic Behavior of Sobolev-Type Orthogonal Polynomials on a Rectifiable Jordan Curve or Arc
}

Amilcar Branquinho, Ana Foulquié Moreno, and Francisco Marcellán

Abstract. Our object of study is the asymptotic behavior of the sequence of polynomials orthogonal with respect to the discrete Sobolev inner product

$$
\langle f, g\rangle=\int_{E} f(\xi) \overline{g(\xi)} \rho(\xi)|d \xi|+f(Z) A g(Z)^{H},
$$

where $E$ is a rectifiabl Jordan curve or arc in the complex plane

$$
f(Z)=\left(f\left(z_{1}\right), \ldots, f^{\left(l_{1}\right)}\left(z_{1}\right), \ldots, f\left(z_{m}\right), \ldots, f^{\left(l_{m}\right)}\left(z_{m}\right)\right),
$$

$A$ is an $M \times M$ Hermitian matrix, $M l_{1}+\cdots+l_{m}+m,|d \xi|$ denotes the arc length measure, $\rho$ is a nonnegative function on $E$, and $z_{i} \in \Omega, i=1,2, \ldots, m$, where $\Omega$ is the exterior region to $E$.

\section{Introduction and Statement of Main Results}

The asymptotic behavior of the polynomial sequence $\left\{P_{n}\right\}_{n \geq 0}$, where $P_{n}(z)=z^{n}+$ lower-degree terms, are orthogonal on $E$, an infinit set of the complex plane, with respect to the measure $\rho(\xi)|d \xi|$, i.e.,

$$
\int_{E} P_{n}(\xi) \bar{\xi}^{k} \rho(\xi)|d \xi|=0, \quad k=0,1, \ldots, n-1,
$$

where $\rho(\xi)$ is a weight function (nonnegative and real-valued), has been analyzed in the works of Korovkin [11] and Suetin [21], where $E$ is a rectifiabl Jordan curve. If $E$ is a set of the complex plane define by the finit union of rectifiabl Jordan arcs and curves, such a question has been studied by Widom [24]. Later on, Aptekarev [2] gave these asymptotic formulas in a more explicit way. In [24], Widom deals with the extremal property of the polynomials $P_{n}$ :

$$
\int_{E}\left|P_{n}(\xi)\right|^{2} \rho(\xi)|d \xi|=\min _{T_{n}=z^{n}+\cdots} \int_{E}\left|T_{n}(\xi)\right|^{2} \rho(\xi)|d \xi|,
$$

AMS classification: Primary 42C05.

Key words and phrases: Orthogonal polynomials, Sobolev inner products, Asymptotics, Jordan curves. 
and carries out this property to a new family of functions in the functional space $H^{2}(\Omega, \rho)$. We will detail this result but, from now on, in order to simplify the notation and to make the presentation clearer, we will restrict it to the case where $E$ is a rectifiabl Jordan curve or arc.

We will assume that $E$ is a rectifiabl Jordan curve or arc in the complex plane, that $\Omega$ is the unbounded component of the complement of $E$, and that $G$ is the unbounded component of the complement of the unit disk, i.e., $G=\{z \in \mathbf{C}:|z|>1\}$. By the Riemann mapping theorem there exists a conformal representation $\Phi: \Omega \rightarrow G$ such that

$$
\Phi(\infty)=\infty \quad \text { and } \quad \lim _{z \rightarrow \infty} \frac{\Phi(z)}{z}=\frac{1}{C(E)}>0,
$$

where $C(E)$ is the capacity of the compact set $E$. These two conditions characterize the function $\Phi$. Let $\Psi: G \rightarrow \Omega$ be the inverse function of $\Phi$. If $E$ is a rectifiabl curve, we can extend $\Phi$ by a nontangential limit to $E$ and, in this case, we get $|\Phi(\xi)|=1$ for $\xi \in E$. In fact, $\left.\Phi\right|_{E}$ is a bijection between $E$ and the unit circle. When $E$ is a rectifiabl arc we denote, by $\Phi_{+}(\xi)$ and $\Phi_{-}(\xi)$, the boundary values on both sides of the arc.

We will denote by $H^{2}(G)$ the Hardy space of analytic functions in $G$, such that

$$
\sup _{1<r<\infty} \int_{D_{r}}|f(z)|^{2}|d z|<\infty,
$$

where $D_{r}=\{z \in \mathbf{C}:|z|=r\}$. This space of functions has been deeply studied in [10]. If the weight function $\rho(\xi)$ satisfie the Szegö condition

$$
\int_{E} \log \rho(\xi)\left|\Phi^{\prime}(\xi)\right||d \xi|>-\infty
$$

we can defin the so-called Szegö function $D_{\rho}$ by the following conditions:

(a) $D_{\rho}$ is analytic in $\Omega, D_{\rho}(z) \neq 0$ in $\Omega$, and $D_{\rho}(\infty)>0$.

(b) There exist boundary values almost everywhere and it holds that

$$
\left|D_{\rho_{ \pm}}(\xi)\right|^{-2}\left|\Phi_{ \pm}^{\prime}(\xi)\right|=\rho(\xi) .
$$

Note that if $E$ is a curve $D_{\rho_{-}}$makes no sense and, in this case, we only refer to $D_{\rho_{+}}$.

Definition 1 (cf. [24]). A function $f$ belongs to the Hardy space $H^{2}(\Omega, \rho)$ if $f$ is analytic in $\Omega$ and if $\left.f \circ \Psi\right|_{D_{\rho \circ \Psi}}$ belongs to $H^{2}(G)$.

Every function $f \in H^{2}(\Omega, \rho)$ has boundary values, $f_{+}$and $f_{-}$in $E$, which belong to $L_{2}(\rho)$ (if $E$ is a curve, then we only consider $f_{+}$). We defin the norm in the space $H^{2}(\Omega, \rho)$ by

$$
\|f\|_{H^{2}(\Omega, \rho)}=\left\{\oint_{E}|f(\xi)|^{2} \rho(\xi)|d \xi|\right\}^{1 / 2}
$$

if $E$ is a curve and

$$
\|f\|_{H^{2}(\Omega, \rho)}=\left\{\int_{E}|f(\xi)|^{2} \rho(\xi)|d \xi|\right\}^{1 / 2}
$$


when $E$ is an arc. For the sake of simplicity, in both cases, we will denote by $\|f\|_{H^{2}(\Omega, \rho)}$ the norm in the space $H^{2}(\Omega, \rho)$.

The extremal property of the sequence $\left\{P_{n}\right\}_{n \geq 0}(1)$, can be restated in the following way. If we denote

$$
m_{n}:=\int_{E}\left|P_{n}(\xi)\right|^{2} \rho(\xi)|d \xi|
$$

and $\varphi_{n}:=P_{n} /[C(E) \Phi]^{n}$, then it holds that

$$
\frac{m_{n}}{C(E)^{2 n}}=\int_{E}\left|\varphi_{n}(\xi)\right|^{2} \rho(\xi)|d \xi|=\min _{\tilde{\varphi}_{n}=T_{n} /[C(E) \Phi]^{n}} \int_{E}\left|\tilde{\varphi}_{n}(\xi)\right|^{2} \rho(\xi)|d \xi|,
$$

where $T_{n}$ is a monic polynomial of degree $n$. These functions $\tilde{\varphi}_{n}$ belong to a certain subspace of $H^{2}(\Omega, \rho)$. We can consider the following extremal problem in $H^{2}(\Omega, \rho)$ :

$$
\mu:=\left\|\varphi^{*}\right\|_{H^{2}(\Omega, \rho)}^{2}=\min _{\substack{\tilde{\varphi} \in \in^{2}(\Omega, \rho) \\ \tilde{\varphi}(\infty)=1}}\|\tilde{\varphi}\|_{H^{2}(\Omega, \rho)}^{2} .
$$

This extremal function $\varphi^{*}$ is exactly $D_{\rho} / D_{\rho}(\infty)$ and we want to point out that if $K(z, \infty):=D_{\rho}(z) / \mu$ then, for every $\varphi \in H^{2}(\Omega, \rho)$, it holds that

$$
\varphi(\infty):=\int_{E} \varphi(\xi) \overline{K(\xi, \infty)} \rho(\xi)|d \xi|,
$$

where the integral symbol is taken in both sides, when $E$ is an arc. In [24], one proves the following result:

Theorem 1. Assume that $E \in C^{2+}$ is a rectifiable Jordan curve or arc and that the weight function $\rho(\xi)$ verifies the Szegó condition (2). It holds that

$$
\begin{gathered}
\lim _{n \rightarrow \infty} \frac{m_{n}}{C(E)^{2 n}}=\mu, \\
P_{n}(z)=[C(E) \Phi(z)]^{n} \varphi^{*}(z)\left[1+\varepsilon_{n}(z)\right], \quad z \in \Omega, \\
\int_{E}\left|C(E)^{-n} P_{n}(\xi)-H_{n}(\xi)\right|^{2} \rho(\xi)|d \xi| \rightarrow 0,
\end{gathered}
$$

where

$$
H_{n}(\xi)= \begin{cases}\Phi^{n}(\xi) \varphi^{*}(\xi), & \xi \in E \text { is a curve } \\ \Phi_{+}^{n}(\xi) \varphi_{+}^{*}(\xi)+\Phi_{-}^{n}(\xi) \varphi_{-}^{*}(\xi), & \xi \in E \text { is an arc }\end{cases}
$$

$\mu$ is defined in (3), and $\varepsilon_{n} \rightrightarrows 0$ in $\Omega$.

Notation. The symbol $\rightrightarrows$ means uniform convergence on compact subsets of the indicated region. 
Let us consider now the following discrete Sobolev inner product

$$
\langle f, g\rangle=\int_{E} f(\xi) \overline{g(\xi)} \rho(\xi)|d \xi|+f(Z) A g(Z)^{H},
$$

where $E$ is a rectifiabl Jordan curve or arc in the complex plane

$$
f(Z)=\left(f\left(z_{1}\right), \ldots, f^{\left(l_{1}\right)}\left(z_{1}\right), \ldots, f\left(z_{m}\right), \ldots, f^{\left(l_{m}\right)}\left(z_{m}\right)\right),
$$

$A$ is an $M \times M$ Hermitian positive definit matrix, $M=l_{1}+\cdots+l_{m}+m,|d \xi|$ denotes the arc length, $z_{i} \in \Omega, i=1,2, \ldots, m$, where $\Omega$ is the unbounded component of the complement of $E$ and the weight function $\rho(\xi)$ verifie the Szegö condition. Notice that (7) is well define for every $z_{i} \in \mathbf{C}$. Nevertheless, using (5), it is well known that for any compact set $K \subset \Omega$, it holds that

$$
P_{n}(z) \neq 0, \quad z \in K,
$$

for $n$ sufficientl large. This property is also verifie for $P_{n}^{(k)}$ for $k=0,1, \ldots$ Since our interest is centered on the asymptotic properties of orthogonal polynomials with respect to (7), we will assume the constraints $z_{i} \in \Omega$, in order to consider, for $n$ sufficientl large, a free region of zeros.

In the last few years, algebraic and analytic properties-like zero distribution, asymptotic behavior, recurrence relations, and differential equations, that the polynomials, orthogonal with respect to this kind of inner products satisfy-have been studied (see [15] with more than 100 titles on this subject).

The study of these systems is motivated by the search for efficien algorithms for computing Fourier expansions of a function $f$ in terms of Sobolev orthogonal polynomials, thus getting better approximation results for the function, as well as for their derivatives and for the analysis of new quadrature Gauss formulas.

Because of the positive-definit character of the matrix $A$, there e xists the polynomial sequence, $\left\{Q_{n}(z)=z^{n}+\text { lower-degree terms }\right\}_{n \geq 0}$, orthogonal with respect to (7). The polynomial $Q_{n}$ can be define by the following orthogonality relations:

$$
\left\langle Q_{n}, z^{k}\right\rangle=0, \quad k=0,1, \ldots, n-1 .
$$

In the present paper, we are studying the asymptotic behavior of this sequence of polynomials, $\left\{Q_{n}\right\}_{n \geq 0}$. This problem has been considered before when $E$ is the interval [-1, 1] (see [1], [14], [16]), or when $E$ is the unit circle (see [4], [13]), although in all these works a wider class of measures has been considered.

Our main goal in this paper is to give a unifie approach, containing the preceding cases.

First of all, we want to point out that, similar to that which was done in the standard case, we can consider the polynomial $Q_{n}$ as solution for the extremal problem

$$
\begin{aligned}
\hat{m}_{n} & :=\int_{E}\left|Q_{n}(\xi)\right|^{2} \rho(\xi)|d \xi|+Q_{n}(Z) A Q_{n}(Z)^{H} \\
& =\min _{T_{n}=z^{n}+\cdots} \int_{E}\left|T_{n}(\xi)\right|^{2} \rho(\xi)|d \xi|+T_{n}(Z) A T_{n}(Z)^{H} .
\end{aligned}
$$


Following this idea, Kaliaguine [8], [9], considers this problem when $l_{i}=0$ for $i=1, \ldots, m$, and $A$ is a diagonal matrix, i.e., he studies the asymptotic behavior of the sequence of monic polynomials, orthogonal with respect to a measure with an absolutely continuous part, plus a finit number of mass points. To carry out this study, Kaliaguine deals with a polynomial modificatio of the measure $\rho(\xi)|d \xi|$, in order to get rid of the masses and, in this way, he recovered the case studied by Widom. In our case, this technique cannot be applied in a natural way because the derivatives, and the nondiagonal structure of the matrix $A$, make the analysis of the situation much more difficult We carry over the technique developed in [4], although in this case we do not have a Christoffel-Darboux formula for the kernel polynomials.

Let $\left\{p_{n}\right\}_{n \geq 0}$, with $p_{n}(z)=k_{n} z^{n}+$ lower-degree terms, $k_{n}>0$, be the sequence of orthonormal polynomials with respect to $\rho(\xi)|d \xi|$, i.e.,

$$
\int_{E} p_{n}(\xi) \overline{p_{m}(\xi)} \rho(\xi)|d \xi|= \begin{cases}0, & n \neq m \\ 1, & n=m\end{cases}
$$

with $k_{n}=m_{n}^{-1 / 2}$ and let

$$
K_{n}(z, w)=\sum_{k=0}^{n-1} p_{k}(z) \overline{p_{k}(w)}
$$

be the kernel polynomials associated to $\rho(\xi)|d \xi|$. We denote that

$$
K_{n}^{(i, j)}(z, w)=\sum_{k=0}^{n-1} p_{k}^{(i)}(z) \overline{p_{k}^{(j)}(w)}, \quad i, j=0,1, \ldots
$$

If the weight function, $\rho(\xi)$, satisfie the Szegö condition (2), we prove (see Theorem 4 in Section 2) that

$$
\frac{K_{n}^{(i, j)}(z, w)}{p_{n}^{(i)}(z) \overline{p_{n}^{(j)}(w)}} \rightrightarrows \frac{1}{\Phi(z) \overline{\Phi(w)}-1}, \quad z, w \in \Omega, \quad i, j=0,1, \ldots
$$

Let $\left\{q_{n}\right\}_{n \geq 0}$, if $q_{n}(z)=\gamma_{n} z^{n}+$ lower-degree terms, $\gamma_{n}>0$, be the sequence of orthonormal polynomials with respect to (7), then we can state the following result:

Theorem 2. Consider an inner product of type (7) such that $E \in C^{2+}$ is a rectifiable Jordan curve or arc, that $\rho(\xi)$ is an integrable nonnegative real-valued function on $E$ that verifies the Szegó condition (2), and that A is a positive-definite matrix of dimension M. It holds that

$$
\begin{gathered}
\lim _{n \rightarrow \infty} \frac{k_{n}}{\gamma_{n}}=\prod_{i=1}^{m}\left|\Phi\left(z_{i}\right)\right|^{l_{i}+1}, \\
\frac{q_{n}^{(k)}(z)}{p_{n}^{(k)}(z)} \rightrightarrows \prod_{i=1}^{m}\left(\frac{\Phi\left(z_{i}\right)}{\left(\Phi(z)-\Phi\left(z_{i}\right)\right)}\right)^{l_{i}+1}, \quad z \in \Omega, \quad k=0,1, \ldots
\end{gathered}
$$


Using (5), (9), and (10), we get the following result:

Corollary 1. With the conditions of Theorem 2 we have

$$
Q_{n}(z)=[C(E) \Phi(z)]^{n} \psi^{*}(z)\left[1+\varepsilon_{n}(z)\right]
$$

where

$$
\psi^{*}(z):=\prod_{i=1}^{m}\left(\frac{\overline{\Phi\left(z_{i}\right)}\left(\Phi(z)-\Phi\left(z_{i}\right)\right)}{\Phi(z) \overline{\Phi\left(z_{i}\right)}-1}\right)^{l_{i}+1} \varphi^{*}(z)
$$

the function $\varphi^{*}$ is the solution of the extremal problem (3) and $\varepsilon_{n} \rightrightarrows 0$ in $\Omega$.

In the framework of an extremal problem we could deduce (3), (5), and (6) for the usual monic orthogonal polynomials. The result obtained in this corollary can also be viewed in this way. Using (3) and (12) we can also see $\psi^{*}$ as the solution of the extremal problem

$$
\hat{\mu}:=\left\|\psi^{*}\right\|_{H^{2}(\Omega, \rho)}^{2}=\min _{\psi \in \tilde{H}}\|\psi\|_{H^{2}(\Omega, \rho)}^{2},
$$

where $\tilde{H}=\left\{\psi \in H^{2}(\Omega, \rho): \psi(\infty)=1, \psi^{(l)}\left(z_{j}\right)=0,0 \leq l \leq l_{j}, 1 \leq j \leq m\right\}$ and

$$
\hat{\mu}=\prod_{j=1}^{m}\left|\Phi\left(z_{j}\right)\right|^{2\left(l_{j}+1\right)} \mu .
$$

We are reminded that $\mu:=\left\|\varphi^{*}\right\|_{H^{2}(\Omega, \rho)}^{2}$.

The proof of this assertion can be done in a similar way as in [8, Lemma 4.2]. Using (9) and (12), we also get

$$
\lim _{n \rightarrow \infty} \frac{\hat{m}_{n}}{C(E)^{2 n}}=\hat{\mu}
$$

The following theorem completes these results:

Theorem 3. If we suppose that the hypotheses of Theorem 2 hold, then

$$
\lim _{n \rightarrow \infty} \int_{E}\left|C(E)^{-n} Q_{n}(\xi)-\tilde{H}_{n}(\xi)\right|^{2} \rho(\xi)|d \xi|=0,
$$

where $\psi^{*}$ is the extremal function given in (12) and

$$
\tilde{H}_{n}(\xi):= \begin{cases}\Phi^{n}(\xi) \psi^{*}(\xi), & \xi \in E \text { is a curve } \\ \Phi_{+}^{n}(\xi) \psi_{+}^{*}(\xi)+\Phi_{-}^{n}(\xi) \psi_{-}^{*}(\xi), & \xi \in E \text { is an arc. }\end{cases}
$$

In the following section, we give some auxiliary results needed to prove these theorems. In the last section, we include their proofs. 


\section{Auxiliary Results}

First of all, we give the following lemma that we will use to prove (8). In fact, we assume, for the weight function $\rho$, more general conditions than the Szegö condition (2). Also notice that we extend the results obtained in [4] for the unit circle.

Lemma 1. Let $\left\{p_{n}\right\}_{n \geq 0}$ be a sequence of polynomials of degree $n$ with zeros in $C \backslash \Omega$, such that

$$
\frac{p_{n+1}(z)}{p_{n}(z)} \rightrightarrows \Phi(z), \quad z \in \Omega,
$$

where $\Phi$ and $\Omega$ have been introduced before. Then

$$
\frac{p_{n}^{(k+1)}(z)}{n p_{n}^{(k)}(z)} \rightrightarrows \frac{\Phi^{\prime}(z)}{\Phi(z)}, \quad z \in \Omega
$$

Proof. First, we will prove the result in the case $k=0$. As $\left\{p_{n+1} / p_{n}\right\}_{n \geq 0}$ is a sequence of analytic functions in $\Omega$, then by (14):

$$
\left(\frac{p_{n+1}(z)}{p_{n}(z)}\right)^{\prime} \rightrightarrows \Phi^{\prime}(z), \quad z \in \Omega
$$

This is equivalent to

$$
\frac{p_{n+1}(z)}{p_{n}(z)}\left[\frac{p_{n+1}^{\prime}(z)}{p_{n+1}(z)}-\frac{p_{n}^{\prime}(z)}{p_{n}(z)}\right] \rightrightarrows \Phi^{\prime}(z), \quad z \in \Omega .
$$

Then we get

$$
\frac{p_{n+1}^{\prime}(z)}{p_{n+1}(z)}-\frac{p_{n}^{\prime}(z)}{p_{n}(z)} \rightrightarrows \frac{\Phi^{\prime}(z)}{\Phi(z)}, \quad z \in \Omega
$$

This yields

$$
\Re e \frac{p_{n+1}^{\prime}(z)}{p_{n+1}(z)}-\Re e \frac{p_{n}^{\prime}(z)}{p_{n}(z)} \rightrightarrows \Re e \frac{\Phi^{\prime}(z)}{\Phi(z)}, \quad z \in \Omega
$$

and

$$
\Im m \frac{p_{n+1}^{\prime}(z)}{p_{n+1}(z)}-\Im m \frac{p_{n}^{\prime}(z)}{p_{n}(z)} \rightrightarrows \Im m \frac{\Phi^{\prime}(z)}{\Phi(z)}, \quad z \in \Omega,
$$

where $\Re e z$ and $\Im m z$ denote the real and imaginary part of $z$, respectively. Using (15), we get

$$
\frac{\exp \Re e \frac{p_{n+1}^{\prime}(z)}{p_{n+1}(z)}}{\exp \Re e \frac{p_{n}^{\prime}(z)}{p_{n}(z)}} \rightrightarrows \exp \Re e \frac{\Phi^{\prime}(z)}{\Phi(z)}, \quad z \in \Omega
$$


Now if we apply [20, Theorem 3.37], then

$$
\left(\exp \Re e \frac{p_{n}^{\prime}(z)}{p_{n}(z)}\right)^{1 / n} \rightrightarrows \exp \Re e \frac{\Phi^{\prime}(z)}{\Phi(z)}, \quad z \in \Omega,
$$

or, equivalently,

$$
\Re e \frac{p_{n}^{\prime}(z)}{n p_{n}(z)} \rightrightarrows \Re e \frac{\Phi^{\prime}(z)}{\Phi(z)}, \quad z \in \Omega .
$$

For the imaginary part, we proceed in the same way and we get the result for $k=0$. For an arbitrary $k$, we use the identity

$$
\frac{q}{q^{\prime}}\left(\frac{p}{q}\right)^{\prime}=\frac{p^{\prime}}{q^{\prime}}-\frac{p}{q}
$$

that holds for the arbitrary differentiable functions $p, q$.

Theorem 4. If $\rho(\xi)$ is a weight function satisfying the Szegó condition (2), then

$$
\frac{K_{n}^{(i, j)}(z, w)}{p_{n}^{(i)}(z) \overline{p_{n}^{(j)}(w)}} \rightrightarrows \frac{1}{\Phi(z) \overline{\Phi(w)}-1}, \quad z, w \in \Omega, \quad i, j=0,1, \ldots
$$

Proof. First, we prove the statement of the theorem for $i, j=0$. Using (4) and (5) we get

$$
p_{n}(z)=[\Phi(z)]^{n} \frac{\varphi^{*}(z)}{\left[\int_{E}\left|\varphi^{*}(\xi)\right|^{2} \rho(\xi)|d \xi|\right]^{1 / 2}}\left[1+\varepsilon_{n}(z)\right], \quad z \in \Omega,
$$

where $\varepsilon_{n}(z) \rightrightarrows 0$ in $\Omega$. Now, we consider the following identity:

$$
\frac{K_{n}(z, w)}{p_{n}(z) \overline{p_{n}(w)}}=\frac{K_{n}(z, w)}{\sum_{k=0}^{n-1} \Phi(z)^{k} \overline{\Phi(w)^{k}}} \frac{\sum_{k=0}^{n-1} \Phi(z)^{k} \overline{\Phi(w)^{k}}}{\Phi(z)^{n} \overline{\Phi(w)^{n}}} \frac{\Phi(z)^{n} \overline{\Phi(w)^{n}}}{p_{n}(z) \overline{p_{n}(w)}} .
$$

Using (17), we deduce

$$
\frac{\Phi(z)^{n} \overline{\Phi(w)^{n}}}{p_{n}(z) \overline{p_{n}(w)}} \rightrightarrows\left[\frac{\varphi^{*}(z) \overline{\varphi^{*}(w)}}{\int_{E}\left|\varphi^{*}(\xi)\right|^{2} \rho(\xi) d \xi}\right]^{-1}, \quad z, w \in \Omega,
$$

and

$$
\frac{\sum_{k=0}^{n-1} \Phi(z)^{k} \overline{\Phi(w)^{k}}}{\Phi(z)^{n} \overline{\Phi(w)^{n}}} \rightrightarrows \frac{1}{\Phi(z) \overline{\Phi(w)}-1}, \quad z, w \in \Omega .
$$

On the other hand,

$$
\begin{aligned}
K_{n}(z, w) & =\sum_{k=0}^{n-1} p_{k}(z) \overline{p_{k}(w)} \\
& =\frac{\varphi^{*}(z) \overline{\varphi^{*}(w)}}{\int_{E}\left|\varphi^{*}(\xi)\right|^{2} \rho(\xi) d \xi} \sum_{k=0}^{n-1} \Phi(z)^{k} \overline{\Phi(w)^{k}}\left(1+y_{k}(z, w)\right),
\end{aligned}
$$


where we denote

$$
y_{k}(z, w):=\varepsilon_{k}(z)+\overline{\varepsilon_{k}(w)}+\varepsilon_{k}(z) \overline{\varepsilon_{k}(w)} .
$$

So we get

$$
\frac{K_{n}(z, w)}{\sum_{k=0}^{n-1} \Phi(z)^{k} \overline{\Phi(w)^{k}}}=\frac{\varphi^{*}(z) \overline{\varphi^{*}(w)}}{\int_{E}\left|\varphi^{*}(\xi)\right|^{2} \rho(\xi) d \xi}\left[1+\frac{\sum_{k=0}^{n-1} \Phi(z)^{k} \overline{\Phi(w)^{k}} y_{k}(z, w)}{\sum_{k=0}^{n-1} \Phi(z)^{k} \overline{\Phi(w)^{k}}}\right] .
$$

Next, we will prove

$$
\frac{\sum_{k=0}^{n-1} \Phi(z)^{k} \overline{\Phi(w)^{k}} y_{k}(z, w)}{\sum_{k=0}^{n-1} \Phi(z)^{k} \overline{\Phi(w)^{k}}} \rightrightarrows 0, \quad z, w \in \Omega .
$$

Taking into account that

$$
\frac{\sum_{k=0}^{n-1} \Phi(z)^{k} \overline{\Phi(w)^{k}} y_{k}(z, w)}{\sum_{k=0}^{n-1} \Phi(z)^{k} \overline{\Phi(w)^{k}}}=\frac{\sum_{k=0}^{n-1} \Phi(z)^{k} \overline{\Phi(w)^{k}} y_{k}(z, w)}{\Phi(z)^{n} \overline{\Phi(w)^{n}}} \frac{\Phi(z)^{n} \overline{\Phi(w)^{n}}}{\sum_{k=0}^{n-1} \Phi(z)^{k} \overline{\Phi(w)^{k}}},
$$

(18) follows if we prove

$$
\frac{\sum_{k=0}^{n-1} \Phi(z)^{k} \overline{\Phi(w)^{k}} y_{k}(z, w)}{\Phi(z)^{n} \overline{\Phi(w)^{n}}} \rightrightarrows 0, \quad z, w \in \Omega .
$$

Indeed, given $K_{1}, K_{2}$ compact sets in $\Omega$ and $\varepsilon>0$, there exists an $n_{0}$, such that for $z \in K_{1}, w \in K_{2}$, and $n \geq n_{0}$ then $\left|y_{n}(z, w)\right|<\varepsilon$. For $z \in K_{1}$ and $w \in K_{2}$ :

$$
\begin{aligned}
& \frac{\sum_{k=0}^{n-1} \Phi(z)^{k} \overline{\Phi(w)^{k}} y_{k}(z, w)}{\Phi(z)^{n} \overline{\Phi(w)^{n}}} \\
& \quad=\frac{\sum_{k=0}^{n_{0}-1} \Phi(z)^{k} \overline{\Phi(w)^{k}} y_{k}(z, w)}{\Phi(z)^{n} \overline{\Phi(w)^{n}}}+\frac{\sum_{k=n_{0}}^{n-1} \Phi(z)^{k} \overline{\Phi(w)^{k}} y_{k}(z, w)}{\Phi(z)^{n} \overline{\Phi(w)^{n}}} .
\end{aligned}
$$

Then

$$
\begin{aligned}
& \left|\frac{\sum_{k=0}^{n-1} \Phi(z)^{k} \overline{\Phi(w)^{k}} y_{k}(z, w)}{\Phi(z)^{n} \overline{\Phi(w)^{n}}}\right| \\
& \quad \leq \frac{\left|\sum_{k=0}^{n_{0}-1} \Phi(z)^{k} \overline{\Phi(w)^{k}} y_{k}(z, w)\right|}{|\Phi(z)|^{n}|\overline{\Phi(w)}|^{n}}+\frac{\sum_{k=n_{0}}^{n-1}|\Phi(z)|^{k}|\overline{\Phi(w)}|^{k}\left|y_{k}(z, w)\right|}{|\Phi(z)|^{n}|\overline{\Phi(w)}|^{n}} \\
& \quad \leq \frac{M\left(n_{0}\right)}{|\Phi(z)|^{n}|\overline{\Phi(w)}|^{n}}+\varepsilon \frac{\sum_{k=n_{0}}^{n-1}|\Phi(z)|^{k}|\overline{\Phi(w)}|^{k}}{|\Phi(z)|^{n}|\overline{\Phi(w)}|^{n}},
\end{aligned}
$$

where $M\left(n_{0}\right)$ is a positive constant depending on $n_{0}$ :

$$
\limsup _{n \rightarrow \infty}\left|\frac{\sum_{k=0}^{n-1} \Phi(z)^{k} \overline{\Phi(w)^{k}} y_{k}(z, w)}{\Phi(z)^{n} \overline{\Phi(w)^{n}}}\right| \leq \frac{\varepsilon}{|\Phi(z) \Phi(w)|-1} .
$$


Now $\varepsilon$ can be made arbitrarily small and we get the result. For $i=1$ and $j=0$, we use (16), and taking into account $p_{n}(z) / p_{n}^{\prime}(z) \rightrightarrows 0, z \in \Omega$, we get

$$
\frac{K_{n}^{(1,0)}(z, w)}{p_{n}^{\prime}(z) \overline{p_{n}(w)}} \rightrightarrows \frac{1}{\Phi(z) \overline{\Phi(w)-1}} .
$$

In the same way, we get the result for arbitrary $i, j \in \mathbf{N}$.

Theorem 5. Let $\mathbf{K}_{n}$ be the matrix of dimension $M$ :

$$
\left(\begin{array}{ccccccc}
K_{n}\left(z_{1}, z_{1}\right) & \ldots & K_{n}^{\left(l_{1}, 0\right)}\left(z_{1}, z_{1}\right) & \ldots & K_{n}\left(z_{m}, z_{1}\right) & \ldots & K_{n}^{\left(l_{m}, 0\right)}\left(z_{m}, z_{1}\right) \\
K_{n}^{(0,1)}\left(z_{1}, z_{1}\right) & \ldots & K_{n}^{\left(l_{1}, 1\right)}\left(z_{1}, z_{1}\right) & \ldots & K_{n}^{(0,1)}\left(z_{m}, z_{1}\right) & \ldots & K_{n}^{\left(l_{m}, 1\right)}\left(z_{m}, z_{1}\right) \\
\vdots & \ldots & \vdots & \ldots & \vdots & \ldots & \vdots \\
K_{n}^{\left(0, l_{1}\right)}\left(z_{1}, z_{1}\right) & \ldots & K_{n}^{\left(l_{1}, l_{1}\right)}\left(z_{1}, z_{1}\right) & \ldots & K_{n}^{\left(0, l_{1}\right)}\left(z_{m}, z_{1}\right) & \ldots & K_{n}^{\left(l_{m}, l_{1}\right)}\left(z_{m}, z_{1}\right) \\
\vdots & \ldots & \vdots & \ldots & \vdots & \ldots & \vdots \\
\vdots & \ldots & \vdots & \ldots & \vdots & \ldots & \vdots \\
K_{n}\left(z_{1}, z_{m}\right) & \ldots & K_{n}^{\left(l_{1}, 0\right)}\left(z_{1}, z_{m}\right) & \ldots & K_{n}\left(z_{m}, z_{m}\right) & \ldots & K_{n}^{\left(l_{m}, 0\right)}\left(z_{m}, z_{m}\right) \\
K_{n}^{(0,1)}\left(z_{1}, z_{m}\right) & \ldots & K_{n}^{\left(l_{1}, 1\right)}\left(z_{1}, z_{m}\right) & \ldots & K_{n}^{(0,1)}\left(z_{m}, z_{m}\right) & \ldots & K_{n}^{\left(l_{m}, 1\right)}\left(z_{m}, z_{m}\right) \\
\vdots & \ldots & \vdots & \ldots & \vdots & \ldots & \vdots \\
K_{n}^{\left(0, l_{m}\right)}\left(z_{1}, z_{m}\right) & \ldots & K_{n}^{\left(l_{1}, l_{m}\right)}\left(z_{1}, z_{m}\right) & \ldots & K_{n}^{\left(0, l_{m}\right)}\left(z_{m}, z_{m}\right) & \ldots & K_{n}^{\left(l_{m}, l_{m}\right)}\left(z_{m}, z_{m}\right)
\end{array}\right)
$$

If $z_{i} \neq z_{j}, i, j=1, \ldots, m$, this matrix is positive definite for $n \geq M$.

\section{Proof (see [4]).}

Let us consider the following function $g(z, w)=1 / \Phi(z) \overline{\Phi(w)}-1$ and denote

$$
g^{(i, j)}(z, w):=\frac{\partial^{i}}{\partial z^{i}} \frac{\partial^{j}}{\partial w^{j}} \overline{\frac{1}{\Phi(z) \overline{\Phi(w)}-1}} .
$$

Let $F_{m}$ be the following matrix of dimension $M$ :

$$
\left(\begin{array}{ccccccc}
g\left(z_{1}, z_{1}\right) & \ldots & g^{\left(l_{1}, 0\right)}\left(z_{1}, z_{1}\right) & \ldots & g\left(z_{m}, z_{1}\right) & \ldots & g^{\left(l_{m}, 0\right)}\left(z_{m}, z_{1}\right) \\
g^{(0,1)}\left(z_{1}, z_{1}\right) & \ldots & g^{\left(l_{1}, 1\right)}\left(z_{1}, z_{1}\right) & \ldots & g^{(0,1)}\left(z_{m}, z_{1}\right) & \ldots & g^{\left(l_{m}, 1\right)}\left(z_{m}, z_{1}\right) \\
\vdots & & \vdots & & \vdots & & \vdots \\
g^{\left(0, l_{1}\right)}\left(z_{1}, z_{1}\right) & \ldots & g^{\left(l_{1}, l_{1}\right)}\left(z_{1}, z_{1}\right) & \ldots & g^{\left(0, l_{1}\right)}\left(z_{m}, z_{1}\right) & \ldots & g^{\left(l_{m}, l_{1}\right)}\left(z_{m}, z_{1}\right) \\
\vdots & & \vdots & & \vdots & & \vdots \\
g\left(z_{1}, z_{m}\right) & \ldots & g^{\left(l_{1}, 0\right)}\left(z_{1}, z_{m}\right) & \ldots & g\left(z_{m}, z_{m}\right) & \ldots & g^{\left(l_{m}, 0\right)}\left(z_{m}, z_{m}\right) \\
g^{(0,1)}\left(z_{1}, z_{m}\right) & \ldots & g^{\left(l_{1}, 1\right)}\left(z_{1}, z_{m}\right) & \ldots & g^{(0,1)}\left(z_{m}, z_{m}\right) & \ldots & g^{\left(l_{m}, 1\right)}\left(z_{m}, z_{m}\right) \\
\vdots & & \vdots & & \vdots & & \vdots \\
g^{\left(0, l_{m}\right)}\left(z_{1}, z_{m}\right) & \ldots & g^{\left(l_{1}, l_{m}\right)}\left(z_{1}, z_{m}\right) & \ldots & g^{\left(0, l_{m}\right)}\left(z_{m}, z_{m}\right) & \ldots & g^{\left(l_{m}, l_{m}\right)}\left(z_{m}, z_{m}\right)
\end{array}\right) .
$$


This matrix can be described by blocks. The $(r, s)$ block is an $\left(l_{r}+1\right) \times\left(l_{s}+1\right)$ matrix

$$
\left(g^{(j, i)}\left(z_{s}, z_{r}\right)\right)_{i=0, \ldots, l_{r}}^{j=0, \ldots, l_{s}},
$$

where $r, s=1, \ldots, m$.

Theorem 6. The matrix $F_{m}$ defined in (20) is nonsingular.

Proof (see [4]). Supposing that $\left|F_{m}\right|=0$, the linear dependence of the rows of the matrix $F_{m}$ is equivalent to the existence of $c_{i j} \in \mathbf{C}, i=1, \ldots, m, j=0, \ldots, l_{i}$, such that for the function

$$
f(z)=\sum_{j=0}^{l_{1}} c_{1, j} g^{(0, j)}\left(z, z_{1}\right)+\cdots+\sum_{j=0}^{l_{m}} c_{m, j} g^{(0, j)}\left(z, z_{m}\right) \not \equiv 0,
$$

where $z_{i}$ is a zero of multiplicity at least $l_{i}+1$. Therefore $f \circ \Phi^{-1}$ has at each $\Phi\left(z_{i}\right)$ a zero of multiplicity at least $l_{i}+1$. Thus, it has at least $M$ zeros, taking into account the multiplicity. But it is immediatly verifiabl that $f\left(\Phi^{-1}(z)\right)=P(z) / Q(z)$, where $P$ is a polynomial of degree at most $M-1$ and $Q$ is a polynomial of degree $M$. This leads us to a contradiction.

Lemma 2. Let $Q$ be an $M \times M$ nonsingular matrix, and $u, x$ two $M$-column vectors. The following identity holds:

$$
1-x^{T} Q^{-1} u=\frac{\operatorname{det}\left[Q-u x^{T}\right]}{\operatorname{det} Q} .
$$

Proof (see [4]).

To conclude this section, we include the following lemma that appears in [8, Lemma 2.2]:

Lemma 3. Let $\left\{f_{n}\right\}_{n \geq 0}$ be a sequence of functions in $H^{2}(\Omega, \rho)$ such that

$$
f_{n}(z) \rightrightarrows f(z), \quad z \in \Omega \quad \text { and } \quad\left\|f_{n}\right\|_{H^{2}(\Omega, \rho)} \leq C, \quad n=0,1, \ldots,
$$

where $C$ is a positive constant. Then

$$
f \in H^{2}(\Omega, \rho) \quad \text { and } \quad\|f\|_{H^{2}(\Omega, \rho)} \leq \liminf _{n \rightarrow \infty}\left\|f_{n}\right\|_{H^{2}(\Omega, \rho)} .
$$

\section{Proof of the Main Results}

Notation. For any function $f(z)$ of one variable and for the vector

$$
X=\left(x_{1,0}, \ldots, x_{1, l_{1}}, \ldots, x_{m, 0}, \ldots, x_{m, l_{m}}\right)
$$


we denote

$$
f(X):=\left(f\left(x_{1,0}\right), \ldots, f^{\left(l_{1}\right)}\left(x_{1, l_{1}}\right), \ldots, f\left(x_{m, 0}\right), \ldots, f^{\left(l_{m}\right)}\left(x_{m, l_{m}}\right)\right) .
$$

Since $K_{n}(w, z)=\overline{K_{n}(z, w)}$ is a function in the variable $w$, then for

$$
Z=(\underbrace{z_{1}, \ldots, z_{1}}_{l_{1}+1}, \ldots, \underbrace{z_{m}, \ldots, z_{m}}_{l_{m}+1})
$$

we write

$$
K_{n}(z, Z)=\overline{K_{n}(Z, z)},
$$

i.e.,

$$
K_{n}(z, Z)=\left(K_{n}\left(z, z_{1}\right), \ldots, K_{n}^{\left(0, l_{1}\right)}\left(z, z_{1}\right), \ldots, K_{n}\left(z, z_{m}\right), \ldots, K_{n}^{\left(0, l_{m}\right)}\left(z, z_{m}\right)\right) .
$$

Notice that $K_{n}^{(i, j)}(z, w)$ means $\sum_{k=0}^{n-1} p_{k}^{(i)}(z) \overline{p_{k}^{(j)}(w)}$. Thus

$$
K_{n}^{(i)}(z, Z)=\left(K_{n}^{(i, 0)}\left(z, z_{1}\right), \ldots, K_{n}^{\left(i, l_{1}\right)}\left(z, z_{1}\right), \ldots,\left(K_{n}^{(i, 0)}\left(z, z_{m}\right), \ldots, K_{n}^{\left(i, l_{m}\right)}\left(z, z_{m}\right)\right) .\right.
$$

First of all, we are going to deduce some algebraic expressions that we will use later to prove Theorem 2 (see [4]).

We expand $q_{n}$ in terms of $\left\{p_{j}\right\}_{j \geq 0}$ :

$$
q_{n}(z)=\frac{\gamma_{n}}{k_{n}} p_{n}(z)+\sum_{k=0}^{n-1} a_{k, n} p_{k}(z)
$$

where

$$
a_{k, n}=\int_{0}^{2 \pi} q_{n}\left(e^{i \theta}\right) \overline{p_{k}\left(e^{i \theta}\right)} d \mu(\theta)=-q_{n}(Z) A p_{k}(Z)^{H}, \quad k=0,1, \ldots, n-1 .
$$

Substituting this expression in (21) we get

$$
\begin{aligned}
q_{n}(z) & =\frac{\gamma_{n}}{k_{n}} p_{n}(z)-q_{n}(Z) A \sum_{k=0}^{n-1} p_{k}(Z)^{H} p_{k}(z) \\
& =\frac{\gamma_{n}}{k_{n}} p_{n}(z)-q_{n}(Z) A K_{n}(z, Z)^{T} .
\end{aligned}
$$

Now we take successive derivatives in (23) and we substitute $z=z_{1}, \ldots, z=z_{m}$, in order to eliminate $q_{n}(Z)$.

Thus

$$
q_{n}(Z)=\frac{\gamma_{n}}{k_{n}} p_{n}(Z)-q_{n}(Z) A \mathbf{K}_{n},
$$

where $\mathbf{K}_{n}$ is the matrix define in (19). 
From (23) we obtain

$$
q_{n}(Z)\left[I_{M}+A \mathbf{K}_{n}\right]=\frac{\gamma_{n}}{k_{n}} p_{n}(Z)
$$

where $I_{M}$ denotes the identity matrix of dimension $M$. Since $\mathbf{K}_{n}$ is a positive definit matrix (see Theorem 5) and the matrix $A$ is positive definite then it holds that the matrix $I_{M}+A \mathbf{K}_{n}$ is a nonsingular matrix. Thus

$$
q_{n}(Z)=\frac{\gamma_{n}}{k_{n}} p_{n}(Z)\left[I_{M}+A \mathbf{K}_{n}\right]^{-1}
$$

Substituting this expression in (23), multiplying by $k_{n} / \gamma_{n}$, and dividing by $p_{n}(z)$, we obtain

$$
\frac{k_{n}}{\gamma_{n}} \frac{q_{n}(z)}{p_{n}(z)}=1-p_{n}(Z)\left[I_{M}+A \mathbf{K}_{n}\right]^{-1} A{\frac{K_{n}(z, Z)^{T}}{p_{n}(z)}}^{T}
$$

On the other hand, from

$$
\left\langle q_{n}, p_{n}\right\rangle=\int_{E} q_{n}(\xi) \overline{p_{n}(\xi)} \rho(\xi)|d \xi|+q_{n}(Z) A p_{n}(Z)^{H}
$$

we get

$$
\frac{k_{n}}{\gamma_{n}}=\frac{\gamma_{n}}{k_{n}}+q_{n}(Z) A p_{n}(Z)^{H} .
$$

Now, multiplying by $k_{n} / \gamma_{n}$ and substituting $q_{n}(Z)$, we obtain

$$
\left(\frac{k_{n}}{\gamma_{n}}\right)^{2}=1+p_{n}(Z)\left[I_{M}+A \mathbf{K}_{n}\right]^{-1} A p_{n}(Z)^{H} .
$$

We are going to express (24) and (25) as a ratio of determinants by using Lemma 2. It holds

$$
\begin{aligned}
\frac{k_{n}}{\gamma_{n}} \frac{q_{n}(z)}{p_{n}(z)} & =\frac{\operatorname{det}\left[I_{M}+A \mathbf{K}_{n}-A{\left.\frac{K_{n}(z, Z)^{T}}{p_{n}(z)} p_{n}(Z)\right]}_{\operatorname{det}\left[I_{M}+A \mathbf{K}_{n}\right]}\right.}{\left(\frac{k_{n}}{\gamma_{n}}\right)^{2}}=\frac{\operatorname{det}\left[I_{M}+A \mathbf{K}_{n}+A p_{n}(Z)^{T} p_{n}(Z)\right]}{\operatorname{det}\left[I_{M}+A \mathbf{K}_{n}\right]},
\end{aligned}
$$

$$
\left(\frac{k_{n}}{\gamma_{n}}\right)^{2}=\frac{\operatorname{det}\left[I_{M}+A \mathbf{K}_{n+1}\right]}{\operatorname{det}\left[I_{M}+A \mathbf{K}_{n}\right]}
$$

Now, using (26) and (27), we can deduce the asymptotic behavior of $k_{n} / \gamma_{n}$ and $q_{n}(z) /$ $p_{n}(z)$ for $z \in \Omega$. 
Proof of Theorem 2. First, we will fin the asymptotic behavior of $k_{n} / \gamma_{n}$ :

$$
\lim _{n \rightarrow \infty}\left(\frac{k_{n}}{\gamma_{n}}\right)^{2}=\lim _{n \rightarrow \infty} \frac{\operatorname{det}\left[A^{-1}+\mathbf{K}_{n+1}\right]}{\operatorname{det}\left[A^{-1}+\mathbf{K}_{n}\right]} .
$$

We introduce the diagonal matrix

$$
\Lambda_{n}=\operatorname{diag}\left(\frac{1}{p_{n}\left(z_{1}\right)}, \frac{1}{p_{n}^{\prime}\left(z_{1}\right)}, \ldots, \frac{1}{p_{n}^{\left(l_{1}\right)}\left(z_{1}\right)}, \ldots, \frac{1}{p_{n}\left(z_{m}\right)}, \frac{1}{p_{n}^{\prime}\left(z_{m}\right)}, \ldots, \frac{1}{p_{n}^{\left(l_{m}\right)}\left(z_{m}\right)}\right) .
$$

Notice that when the measure of orthogonality is in Szegő class we can ensure, using (5), that this matrix is well defined for $n$ sufficientl large. Then we get

$$
\lim _{n \rightarrow \infty}\left(\frac{k_{n}}{\gamma_{n}}\right)^{2}=\lim _{n \rightarrow \infty} \frac{\operatorname{det}\left[\overline{\Lambda_{n+1}} A^{-1} \Lambda_{n+1}+\overline{\Lambda_{n+1}} \mathbf{K}_{n+1} \Lambda_{n+1}\right]}{\operatorname{det}\left[\overline{\Lambda_{n}} A^{-1} \Lambda_{n}+\overline{\Lambda_{n}} \mathbf{K}_{n} \Lambda_{n}\right]} \frac{\operatorname{det}\left[\overline{\Lambda_{n}} \Lambda_{n}\right]}{\operatorname{det}\left[\overline{\Lambda_{n+1}} \Lambda_{n+1}\right]} .
$$

The matrix $\overline{\Lambda_{n}} \mathbf{K}_{n} \Lambda_{n}$ can be described by blocks. The $(r, s)$ block is an $\left(l_{r}+1\right) \times\left(l_{s}+1\right)$ matrix

$$
\left(\frac{K_{n}^{(j, i)}\left(z_{s}, z_{r}\right)}{p_{n}^{(j)}\left(z_{s}\right) \overline{p_{n}^{(i)}\left(z_{r}\right)}}\right)_{i=0, \ldots, l_{r}}^{j=0, \ldots, l_{s}},
$$

where $r, s=1, \ldots, m$. Using Theorem 4 and relation (5), we conclude that

$$
\lim _{n \rightarrow \infty} \operatorname{det}\left[\overline{\Lambda_{n+1}} A^{-1} \Lambda_{n+1}+\overline{\Lambda_{n+1}} \mathbf{K}_{n+1} \Lambda_{n+1}\right]=0
$$

and we need to compute a limit of the form $0 / 0$ (if $l_{j}=0$ for $j=0, \ldots, m$, as we do not have this problem, we can compute the limit, nevertheless, this case has been treated in [8] and [9]). In [7], we fin a similar situation for a system of equations. Here, we adapt some ideas that appear in similar works.

For all $f, h$ differentiable functions and $v=0,1,2, \ldots$, it holds that

$$
\frac{f^{(v)}}{h^{(v)}}=\left(\frac{f}{h}\right)^{(v)} \frac{h}{h^{(v)}}-\sum_{k=1}^{\nu} F(v, k) \frac{f^{(v-k)}}{h^{(v-k)}},
$$

where

$$
F(v, k)=\left(\begin{array}{l}
v \\
k
\end{array}\right) \frac{h h^{(\nu-k)}}{h^{(v)}}\left(\frac{1}{h}\right)^{(k)} .
$$

Notice that the coefficient $F(v, k)$ do not depend on the function $f$. If we take $f=h$, we get the following relation:

$$
1+\sum_{k=1}^{\nu} F(\nu, k)=0
$$

Now, in

$$
\operatorname{det}\left[\overline{\Lambda_{n+1}} A^{-1} \Lambda_{n+1}+\overline{\Lambda_{n+1}} \mathbf{K}_{n+1} \Lambda_{n+1}\right]
$$


add to the $\sum_{p=1}^{s-1}\left(l_{p}+1\right)+1+k$ row, for $1 \leq k \leq l_{s}$ and $1 \leq s \leq m$, a linear combination of the preceding $k-1$ rows with coefficient $F(v, k)$ :

$$
h(z):=\overline{p_{n+1}(z)}
$$

and $z=z_{s}$. Then, multiply the resulting row by

$$
\overline{h^{(k)}(z) / h(z)}
$$

evaluated at $z=z_{s}$. We also carry out this kind of elementary operation by rows with

$$
\operatorname{det}\left[\overline{\Lambda_{n}} A^{-1} \Lambda_{n}+\overline{\Lambda_{n}} \mathbf{K}_{n} \Lambda_{n}\right]
$$

where, in this case,

$$
h(z):=\overline{p_{n}(z)}
$$

On doing these elementary operations by rows, we fin that

$$
\begin{aligned}
\frac{\operatorname{det}\left[\overline{\Lambda_{n+1}}\right.}{\left.A^{-1} \Lambda_{n+1}+\overline{\Lambda_{n+1}} \mathbf{K}_{n+1} \Lambda_{n+1}\right]} & \\
\operatorname{det}\left[\overline{\Lambda_{n}} A^{-1} \Lambda_{n}+\overline{\Lambda_{n}} \mathbf{K}_{n} \Lambda_{n}\right] & \\
= & \frac{\prod_{j=1}^{m} \prod_{s=1}^{l_{j}} \frac{\overline{p_{n}^{(s)}\left(z_{j}\right)}}{p_{n}\left(z_{j}\right)} \operatorname{det}\left[B_{n+1}+H_{n+1}\right]}{\prod_{j=1}^{m} \prod_{s=1}^{l_{j}} \overline{p_{n+1}^{(s)}\left(z_{j}\right)}} \operatorname{det}\left[B_{n}+H_{n}\right]
\end{aligned}
$$

Here $B_{n}$ is a matrix, which can be described by blocks. The $(r, s)$ block is the $\left(l_{r}+1\right) \times$ $\left(l_{s}+1\right)$ matrix

$$
\left(\left[\frac{b_{i, j}^{(r, s)}}{p_{n}^{(j)}\left(z_{s}\right) \overline{p_{n}^{(i)}\left(z_{r}\right)}}+\sum_{k=1}^{i} F(i, k) \frac{b_{i-k, j}^{(r, s)}}{p_{n}^{(j)}\left(z_{s}\right) \overline{p_{n}^{(i-k)}\left(z_{r}\right)}}\right] \overline{\frac{p_{n}^{(i)}\left(z_{r}\right)}{p_{n}\left(z_{r}\right)}}\right)_{i=0, \ldots, l_{r}}^{j=0, \ldots, l_{s}},
$$

where $b_{i, j}^{(r, s)}$ are constants

$$
F(i, k)=\left.\left(\begin{array}{l}
i \\
k
\end{array}\right) \overline{p_{n}\left(z_{r}\right) \frac{p_{n}^{(i-k)}\left(z_{r}\right)}{p_{n}^{(i)}\left(z_{r}\right)}\left(\frac{1}{p_{n}(z)}\right)^{(k)}}\right|_{z=z_{r}} .
$$

Also, $H_{n}$ is a matrix which can be described by blocks. The $(r, s)$ block is the $\left(l_{r}+1\right) \times$ $\left(l_{s}+1\right)$ matrix

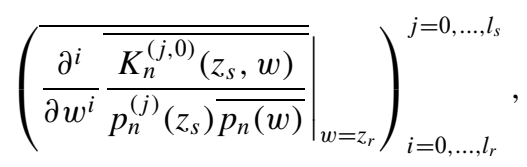


where $r, s=1, \ldots, m$. Notice that

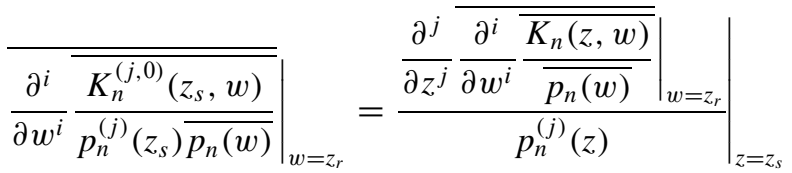

for $1 \leq r, s \leq m, 0 \leq j \leq l_{s}$, and $0 \leq i \leq l_{r}$.

Before we can fin the limit in (30), as $n$ tends to infinit, we have to carry out transformations similar to those above, but by columns on the determinants

$$
\operatorname{det}\left[B_{n+1}+H_{n+1}\right] \quad \text { and } \quad \operatorname{det}\left[B_{n}+H_{n}\right] \text {. }
$$

We describe these elementary operations on $\operatorname{det}\left[B_{n+1}+H_{n+1}\right]$. Those corresponding to $\operatorname{det}\left[B_{n}+H_{n}\right]$ are the same with $n+1$ substituted by $n$.

Let $1 \leq k \leq l_{s}$ and $1 \leq s \leq m$. Add to the $\sum_{p=1}^{s-1}\left(l_{p}+1\right)+1+k$ column of $\operatorname{det}\left[B_{n+1}+H_{n+1}\right]$ a linear combination of the preceding $k-1$ columns with the coefficient define in (28) with

$$
h(z):=p_{n+1}(z)
$$

evaluated at $z=z_{s}$ and then multiply the resulting column by

$$
h^{(k)}(z) / h(z) \text { evaluated at } z=z_{s} \text {. }
$$

After carrying out similar operations on $\operatorname{det}\left[B_{n}+H_{n}\right]$, we fin that

$$
\begin{gathered}
\prod_{j=1}^{m} \prod_{s=1}^{l_{j}} \overline{\frac{p_{n}^{(s)}\left(z_{j}\right)}{p_{n}\left(z_{j}\right)}} \operatorname{det}\left[B_{n+1}+H_{n+1}\right] \\
\prod_{j=1}^{m} \prod_{s=1}^{l_{j}} \overline{\frac{p_{n+1}^{(s)}\left(z_{j}\right)}{p_{n+1}\left(z_{j}\right)}} \operatorname{det}\left[B_{n}+H_{n}\right] \\
=\frac{\prod_{j=1}^{m} \prod_{s=1}^{l_{j}}\left|\frac{p_{n}^{(s)}\left(z_{j}\right)}{p_{n}\left(z_{j}\right)}\right|^{2}}{\prod_{j=1}^{m} \prod_{s=1}^{l_{j}}\left|\frac{p_{n+1}^{(s)}\left(z_{j}\right)}{p_{n+1}\left(z_{j}\right)}\right|^{2}} \frac{\operatorname{det}\left[C_{n+1}+R_{n+1}\right]}{\operatorname{det}\left[C_{n}+R_{n}\right]},
\end{gathered}
$$

where $C_{n}$ is a block matrix. The $(r, s)$ block is the $\left(l_{r}+1\right) \times\left(l_{s}+1\right)$ matrix whose $(i, j)$ entry for $i=0, \ldots, l_{r}$ and $j=0, \ldots, l_{s}$ is

$$
\begin{aligned}
& \frac{p_{n}^{(j)}\left(z_{s}\right)}{p_{n}\left(z_{s}\right)}\left[\left[\frac{b_{i, j}^{(r, s)}}{p_{n}^{(j)}\left(z_{s}\right) \overline{p_{n}^{(i)}\left(z_{r}\right)}}+\sum_{k=1}^{i} F(i, k) \frac{b_{i-k, j}^{(r, s)}}{p_{n}^{(j)}\left(z_{s}\right) \overline{p_{n}^{(i-k)}\left(z_{r}\right)}}\right] \overline{\frac{p_{n}^{(i)}\left(z_{r}\right)}{p_{n}\left(z_{r}\right)}}\right. \\
& \left.+\sum_{u=1}^{j} \tilde{F}(j, u)\left[\frac{b_{i, j-u}^{(r, s)}}{p_{n}^{(j-u)}\left(z_{s}\right) \overline{p_{n}^{(i)}\left(z_{r}\right)}}+\sum_{k=1}^{i} F(i, k) \frac{b_{i-k, j-u}^{(r, s)}}{p_{n}^{(j-u)}\left(z_{s}\right) \overline{p_{n}^{(i-k)}\left(z_{r}\right)}}\right] \overline{\frac{p_{n}^{(i)}\left(z_{r}\right)}{p_{n}\left(z_{r}\right)}}\right],
\end{aligned}
$$


where

$$
\tilde{F}(j, u)=\left(\begin{array}{l}
j \\
u
\end{array}\right) p_{n}\left(z_{s}\right) \frac{p_{n}^{(j-u)}\left(z_{s}\right)}{p_{n}^{(j)}\left(z_{s}\right)}\left(\frac{1}{p_{n}\left(z_{s}\right)}\right)^{(u)},
$$

and $F(i, k)$ is given by (31). Notice that the elements of the matrix $C_{n}$ are $o(1)$, and $R_{n}$ is a block matrix. The $r, s$ block is the $\left(l_{r}+1\right) \times\left(l_{s}+1\right)$ matrix

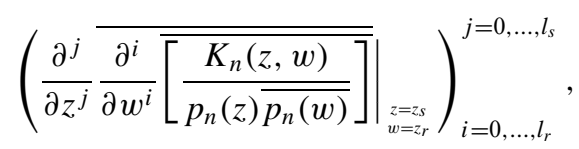

where $r, s=1, \ldots, m$. Taking into account Theorem 4, we obtain

$$
\lim _{n \rightarrow \infty} \operatorname{det}\left[C_{n}+R_{n}\right]=\lim _{n \rightarrow \infty} \operatorname{det}\left[o(1)+R_{n}\right]=\left|F_{m}\right| \neq 0,
$$

where $F_{m}$ is the matrix define in (20). From this, we have

$$
\lim _{n \rightarrow \infty} \frac{k_{n}}{\gamma_{n}}=\prod_{i=1}^{m}\left|\Phi\left(z_{i}\right)\right|^{l_{i}+1}
$$

Using similar arguments, we can obtain the asymptotic behavior of $q_{n}(z) / p_{n}(z)$. On account of (26) and (9), this is restricted to findin the limit of

$$
\begin{aligned}
\frac{\operatorname{det}\left[A^{-1}+\mathbf{K}_{n}-\frac{K_{n}(z, Z)^{T}}{p_{n}(z)} p_{n}(Z)\right]}{\operatorname{det}\left[A^{-1}+\mathbf{K}_{n}\right]} \\
=\frac{\operatorname{det}\left[\overline{\Lambda_{n}} A^{-1} \Lambda_{n}+\overline{\Lambda_{n}} \mathbf{K}_{n} \Lambda_{n}-\overline{\Lambda_{n}} \frac{K_{n}(z, Z)^{T}}{p_{n}(z)} p_{n}(Z) \Lambda_{n}\right]}{\operatorname{det}\left[\overline{\Lambda_{n}} A^{-1} \Lambda_{n}+\overline{\Lambda_{n}} \mathbf{K}_{n} \Lambda_{n}\right]} .
\end{aligned}
$$

In

$$
\operatorname{det}\left[\overline{\Lambda_{n}} A^{-1} \Lambda_{n}+\overline{\Lambda_{n}} \mathbf{K}_{n} \Lambda_{n}-\overline{\Lambda_{n}}{\frac{K_{n}(z, Z)}{p_{n}(z)}}^{T} p_{n}(Z) \Lambda_{n}\right],
$$

add to the $\sum_{p=1}^{s-1}\left(l_{p}+1\right)+1+k$ row, for $1 \leq k \leq l_{s}$ and $1 \leq s \leq m$, a linear combination of the preceding $k-1$ rows with the coefficient define in (28) with

$$
h(z):=\overline{p_{n}(z)}
$$

evaluated at $z=z_{s}$, and multiply the resulting row by

$$
\overline{h^{(k)}(z) / h(z)} \text { evaluated at } z=z_{s} .
$$

The resulting determinant is transformed by columns in a similar way. The same transformations by rows and columns are made on $\operatorname{det}\left[\overline{\Lambda_{n}} A^{-1} \Lambda_{n}+\overline{\Lambda_{n}} \mathbf{K}_{n} \Lambda_{n}\right]$. 
Taking into account (29), we fin that

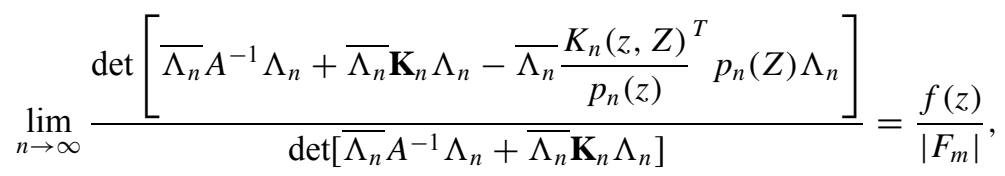

where $F_{m}$ is the matrix define in (20) and $f(z)$ is the determinant of a block matrix whose $(r, s)$ block is the $\left(l_{r}+1\right) \times\left(l_{s}+1\right)$ matrix whose firs column is equal to

$$
\left(\begin{array}{c}
g\left(z_{s}, z_{r}\right)-g\left(z, z_{r}\right) \\
g^{(0,1)}\left(z_{s}, z_{r}\right)-g^{(0,1)}\left(z, z_{r}\right) \\
\vdots \\
g^{\left(0, l_{r}\right)}\left(z_{s}, z_{r}\right)-g^{\left(0, l_{r}\right)}\left(z, z_{r}\right)
\end{array}\right)
$$

and other entries of this matrix are

$$
\left(g^{(i-1, j-1)}\left(z_{s}, z_{r}\right)\right)_{\substack{j=1, \ldots, \ldots, l_{r}+1 \\ j=2, \ldots, l_{s}+1}},
$$

where $r, s=1, \ldots, m$. If we subtract to the $\sum_{s=1}^{i-1}\left(l_{s}+1\right)+1$ column of $f(z)$ its firs column for $i=2, \ldots, m$, we obtain that the dependence on the variable $z$ only appears in the firs column of this determinant. From this, if we defin

$$
p(z):=f\left(\Phi^{-1}(z)\right) \prod_{j=1}^{m}\left(z \overline{\Phi\left(z_{j}\right)}-1\right)^{l_{j}+1}
$$

it follows immediately that $p$ is a polynomial in the variable $z$ of degree at most $\sum_{i=1}^{m}\left(l_{i}+\right.$ 1). Furthermore,

$$
\left.f\left(\Phi^{-1}(z)\right)^{(s)}\right|_{z=\Phi\left(z_{i}\right)}=0, \quad 0 \leq s \leq l_{i}, \quad i=1, \ldots, m .
$$

This yields that

$$
\left.p^{(s)}(z)\right|_{z=\Phi\left(z_{i}\right)}=0, \quad 0 \leq s \leq l_{i}, \quad i=1, \ldots, m .
$$

From this, we deduce that either $p$ is the polynomial identically equal to zero or that there exists a nonzero complex constant $C$ such that

$$
p(z)=C \prod_{i=1}^{m}\left(z-\Phi\left(z_{i}\right)\right)^{l_{i}+1} .
$$

Let us calculate $p^{\left(\Sigma_{i=1}^{m}\left(l_{i}+1\right)\right)}(z)$ using Leibniz's formula on (32). If we take into account the equality

$$
\left(\prod_{j=1}^{m}\left(z \overline{\Phi\left(z_{j}\right)}-1\right)^{l_{j}+1}\right)^{\left(\Sigma_{i=1}^{m}\left(l_{i}+1\right)\right)}=\left(\sum_{i=1}^{m}\left(l_{i}+1\right)\right) ! \prod_{j=1}^{m} \overline{\Phi\left(z_{j}\right)^{l_{j}+1}}
$$


and since

$$
\prod_{j=1}^{m}\left(z \overline{\Phi\left(z_{j}\right)}-1\right)^{l_{j}+1} g^{(0, s)}\left(\Phi^{-1}(z), z_{i}\right), \quad i=1, \ldots, m, \quad s=0, \ldots, l_{i}
$$

is a polynomial in the variable $z$ of degree $\sum_{i=1}^{m}\left(l_{i}+1\right)-1$ (therefore its $\sum_{i=1}^{m}\left(l_{i}+1\right)$ derivative is identically zero), it holds that $p(z)^{\left(\Sigma_{i=1}^{m}\left(l_{i}+1\right)\right)}$ is equal to

$$
\left(\sum_{i=1}^{m}\left(l_{i}+1\right)\right) ! \prod_{j=1}^{m}{\overline{\Phi\left(z_{j}\right)}}^{l_{j}+1}\left|F_{m}\right| \neq 0
$$

Therefore,

$$
f(z)=\prod_{i=1}^{m}\left(\frac{\overline{\Phi\left(z_{i}\right)}\left(\Phi(z)-\Phi\left(z_{i}\right)\right.}{\left(\Phi(z) \overline{\Phi\left(z_{i}\right)}-1\right)}\right)^{l_{i}+1}\left|F_{m}\right|
$$

Now, using (9) and (26), we deduce

$$
\lim _{n \rightarrow \infty} \frac{q_{n}(z)}{p_{n}(z)}=\prod_{i=1}^{m}\left(\frac{\overline{\Phi\left(z_{i}\right)}\left(\Phi(z)-\Phi\left(z_{i}\right)\right)}{\left|\Phi\left(z_{i}\right)\right|\left(\Phi(z) \overline{\Phi\left(z_{i}\right)}-1\right)}\right)^{l_{i}+1}
$$

and we get (10) for $k=0$. For any $k$, the result follows using induction. In this way, we prove Theorem 2.

Proof of Theorem 3. Let $\psi_{n}:=Q_{n} /(C(E) \Phi)^{n}$. From (11), we get

$$
\lim _{n \rightarrow \infty} \psi_{n}^{(j)}\left(z_{i}\right)=0, \quad i=1, \ldots, m, \quad j=0, \ldots, l_{i}
$$

We assume that $E$ is a rectifiabl Jordan curve. It holds that

$$
\left\|\psi_{n}\right\|_{H^{2}(\Omega, \rho)}^{2} \leq \frac{\hat{m}_{n}}{C(E)^{2 n}},
$$

where

$$
\hat{m}_{n}=\int_{E}\left|Q_{n}(\xi)\right|^{2} \rho(\xi)|d \xi|+Q_{n}(Z) A Q_{n}(Z)^{H}
$$

From (13), we get

$$
\limsup _{n \rightarrow \infty}\left\|\psi_{n}\right\|_{H^{2}(\Omega, \rho)}^{2} \leq \hat{\mu}
$$

This implies that the sequence of functions $\left\{\frac{1}{2}\left(\psi_{n}+\psi^{*}\right)\right\}$ in $H^{2}(\Omega, \rho)$ is uniformly bounded in $L^{2}(\rho)$. Using (11), we get

$$
\frac{1}{2}\left(\psi_{n}+\psi^{*}\right) \rightrightarrows \psi^{*} .
$$


Now, from Lemma 3, we can state that

$$
4 \hat{\mu} \leq \liminf _{n \rightarrow \infty}\left\|\psi_{n}+\psi^{*}(z)\right\|_{H^{2}(\Omega, \rho)}^{2} .
$$

Now, using the parallelogram identity,

$$
\limsup _{n \rightarrow \infty}\left\|\psi_{n}(z)-\psi^{*}(z)\right\|_{H^{2}(\Omega, \rho)}^{2} \leq 2 \limsup _{n \rightarrow \infty}\left\|\psi_{n}\right\|_{H^{2}(\Omega, \rho)}^{2}+2\left\|\psi^{*}\right\|_{H^{2}(\Omega, \rho)}^{2}-4 \hat{\mu} \leq 0
$$

and, from this last expression, we deduce the statement of the theorem.

If $E$ is a rectifiabl Jordan arc, then

$$
\begin{aligned}
\int_{E} \mid C(E)^{-n} & Q_{n}(\xi)-\left.\tilde{H}_{n}(\xi)\right|^{2} \rho(\xi)|d \xi| \\
= & \int_{E}\left|C(E)^{-n} Q_{n}(\xi)\right|^{2} \rho(\xi)|d \xi|+\int_{E}\left|\tilde{H}_{n}(\xi)\right|^{2} \rho(\xi)|d \xi| \\
& \quad-2 \Re e \int_{E} C(E)^{-n} Q_{n}(\xi) \overline{\tilde{H}_{n}(\xi)} \rho(\xi)|d \xi| .
\end{aligned}
$$

Let us estimate each te rm of this sum

$$
\begin{gathered}
\int_{E}\left|C(E)^{-n} Q_{n}(\xi)\right|^{2} \rho(\xi)|d \xi|=\frac{\hat{m}_{n}}{C(E)^{2 n}}=\hat{\mu}+o(1), \\
\int_{E}\left|\tilde{H}_{n}(\xi)\right|^{2} \rho(\xi)|d \xi|=\left\|\psi^{*}\right\|_{H^{2}(\Omega, \rho)}^{2}+2 \Re e \int_{E} \overline{\Phi_{+}^{n}(\xi) \psi_{+}^{*}(\xi)} \Phi_{-}^{n}(\xi) \psi_{-}^{*}(\xi) \rho(\xi)|d \xi| .
\end{gathered}
$$

The second term tends to 0 when $n \rightarrow \infty$ (see [24, Lemma 12.1, p. 218]). Thus

$$
\int_{E}\left|\tilde{H}_{n}(\xi)\right|^{2} \rho(\xi)|d \xi|=\hat{\mu}+o(1) .
$$

Let us use the relations $\psi^{*}(z)=B(z) \varphi^{*}(z), \psi^{*}(z)=\mu K(z, \infty)$, where $K$ is the reproducing kernel, $\hat{\mu}=\prod_{j=1}^{m}\left|\Phi\left(z_{j}\right)\right|^{2\left(l_{j}+1\right)} \mu$, and $\overline{\Phi_{+}(\xi)}=1 / \Phi_{+}(\xi)$ for $\xi \in E$, in the following transformations:

$$
\begin{aligned}
2 \Re e \int_{E} C(E)^{-n} Q_{n}(\xi) \overline{\tilde{H}_{n}(\xi)} \rho(\xi)|d \xi| & =2 \Re e \oint_{E} \frac{Q_{n}(\xi)}{C(E)^{n} \Phi^{n}(\xi)} \overline{\psi^{*}(\xi)} \rho(\xi)|d \xi| \\
& =2 \Re e \oint_{E} \psi_{n}(\xi) \mu \overline{B(\xi) K(\xi, \infty)} \rho(\xi)|d \xi| \\
& =2 \mu \Re e \oint_{E} \frac{\psi_{n}(\xi)}{B(\xi)} \mu|B(\xi)|^{2} \overline{K(\xi, \infty)} \rho(\xi)|d \xi| \\
& =2 \hat{\mu} \Re e \oint_{E} \frac{\psi_{n}(\xi)}{B(\xi)} \overline{K(\xi, \infty)} \rho(\xi)|d \xi|
\end{aligned}
$$

From (33),

$$
\frac{\psi_{n}(z)}{B(z)}=\sum_{i=1}^{m} \sum_{k=0}^{l_{i}} \frac{A_{n}^{(i, k)}}{\left(z-z_{i}\right)^{k}}+r_{n}(z)
$$


where $\lim _{n \rightarrow \infty} A_{n}^{(i, k)}=0, r_{n}(\infty)=1, r_{n} \in H^{2}(\Omega, \rho)$. So, using the reproducing property of the kernel, we have

$$
2 \Re e \int_{E} C(E)^{-n} Q_{n}(\xi) \overline{\tilde{H}_{n}(\xi)} \rho(\xi)|d \xi|=2 \hat{\mu}+o(1) .
$$

From this last expre ssion, we get the statement of the theorem.

Acknowledgments. The work of the firs author was supported by the Portuguese Ministry of Science and Technology, Fundaçao para a Ciência e Tecnologiá of Portugal under grant FMRH-BSAB-109-99 and by the Centro de Matemática da Universidade de Coimbra. The second author would also like to thank the Unidade de Investigaçao (Matemática e Aplicaçoes) of the University of Aveiro for their support. The work of the second and third authors was supported by the Dirección General de Ensenanza Superior (DGES) of Spain under grant PB 96-0120-C03-01.

\section{References}

1. M. Alfaro, F. Marcellán, M. L. Rezola, A. RonveauX (1995): Sobolev-type orthogonal polynomials: The nondiagonal case. J. Approx. Theory, 83:266-287.

2. A. I. APTEKAREV (1984): Asymptotic properties of polynomials orthogonal on a system of contours, and periodic motions of Toda lattices. Mat. Sb., 125:167, 231-258; English transl. in Math. USSR-Sb., 53 (1986), 233-260.

3. A. CACHAFEIRO, F. MARCELLÁN (1994): Orthogonal polynomials of Sobolev type on the unit circle. J. Approx. Theory, 78:127-146.

4. A. FoulQuiÉ Moreno, F. MARCELlÁN, K. PAN (1999): Asymptotic behavior of Sobolev-type orthogonal polynomials on the unit circle. J. Approx. Theory, 100:345-363.

5. A. FoUlquiÉ MoRenO, F. MARCELlÁN (1997): Strong asymptotics for polynomials orthogonal with respect to a discrete Sobolev inner product on the support of the measure of orthogonality. Methods Appl. Anal. 4 (1):53-66.

6. A. Foulquié Moreno, F. MARCellán, F. Peherstorfer, R. STEInBAuer (1998): Strong asymptotics on the support of the measure of orthogonality for polynomials orthogonal with respect to a discrete Sobolev inner product on the unit circle. Rend. Circ. Mat. Palermo, Ser. II, Suppl. pp. 411-425.

7. A. A. GONČAR (1975): On convergence of Pade approximants for some classes of meromorphic functions. Math. USSR Sb., 26:555-575.

8. V. A. KALIAGUINE (1995): A note on the asymptotics of orthogonal polynomials on a complex arc: The case of a measure with a discrete part. J. Approx. Theory, 80:138-145.

9. V. A. Kaliaguine, R. Benzine (1989): Sur la formule asymptotique des polynômes orthogonaux associes à une mesure concentree sur un contour plus une partie finie. Bull. Soc. Math. Belg., 41: 29-46.

10. P. Koosis (1980): Introduction to $H_{p}$ Spaces. London Math. Soc. Lecture Notes Series, Vol. 40. Cambridge, UK: Cambridge University Press.

11. P. KOROVKIN (1941): Sur des polynômes orthogonaux sur un contour. Mat. Sb., 9:469-484.

12. X. LI, K. PAN (1994): Asymptotic behavior of orthogonal polynomials corresponding to a measure with discrete part off the unit circle. J. Approx. Theory, 79:54-71.

13. X. LI, F. MARCELLÁN (1996): On polynomials orthogonal with respect to Sobolev inner product on the unit circle. Pacifi J. Math., 175:127-146.

14. G. LÓPEZ, F. MARCELLÁN, W. VAN ASSCHE (1995): Relative asymptotics for polynomials orthogonal with respect to a discrete Sobolev inner product. Constr. Approx., 11:107-137.

15. F. MARCELlÁN, A. RonVEAUX (1995): Orthogonal polynomials and Sobolev inner products. A bibliography. Facultés Universitaires N. D. de la Paix. Namur. 
16. F. MARCELLÁN, W. VAN ASSCHE (1993): Relative asymptotics for orthogonal polynomials with a Sobolev inner product. J. Approx. Theory, 72:193-209.

17. A. Martínez Finkelshtein, T. E. Pérez, J. J. Moreno-Balcázar, M. A. Piñar (1998): Asymptotics of Sobolev orthogonal polynomials for coherent pairs of measures. J. Approx. Theory, 92:280-293.

18. A. MATÉ, P. NEVAI, V. TOTIK (1987): Extensions of Szegö's theory of orthogonal polynomials II. Constr. Approx., 3:51-72.

19. E. A. RAKHMANOV (1983): On the asymptotics of the ratio of orthogonal polynomials II. USSR Sb., 46: 105-117.

20. W. RUDIN (1987): Real and Complex Analysis, 3rd ed. Madrid: McGraw-Hill

21. P. K. SUETIN (1966): Fundamental properties of polynomials orthogonal on a contour. Russian Math. Surveys, 21:35-83.

22. H. Stahl, V. TotiK (1992): General Orthogonal Polynomials. Encyclopedia of Mathematics and its Applications, Vol. 43. Cambridge: Cambridge University Press.

23. G. SzEGÖ (1975): Orthogonal Polynomials. Colloq. Publ., Vol. 23, 4th ed. Providence, RI: American Mathematical Society.

24. H. WIDOM (1969): Extremal polynomials associated with a system of curves in the complex plane, Adv. in Math., 3:123-161.

A. Branquinho
Departamento de Matemática
Universidade de Coimbra
Apartado 3008
3000 Coimbra
Portugal
ajplb@mat.uc.pt

A. Branquinho

Universidade de Coimbra

ajplb@mat.uc.p

A. Foulquié Moreno
Departamento de Matemática
Universidade de Aveiro
Campus de Santiago 3810
Aveiro
Portugal
foulquié@mat.ua.pt

F. Marcellán

Departamento de Matemática

Escuela Politécnica Superior

Universidade Carlos III

C. Butarque, 15

28911 Leganés-Madrid

Spain

pacomarc@ing.uc3m.es 